\title{
División, objeto y abstracción de las ciencias especulativas según Francisco Suárez
}

\author{
(1) José María Felipe Mendoza \\ Universidad de Cuyo - CONICET, Argentina
}

Recibido el o2 de septiembre de 2019. Aceptado el 29 de octubre de 2019.

\begin{abstract}
Resumen
Durante los últimos decenios del siglo XX la filosofía de Francisco Suárez fue considerada parte integrante de un movimiento escolástico comúnmente mencionado como escuela tomista. La perspectiva de abordaje, según el mote impuesto, colocaba la posición especulativa del Dr. Eximio más próxima al dominico Tomás de Aquino de acuerdo con los siguientes matices: a. en el ámbito epistémico la figura del Aquinate eclipsaba ampliamente aquella de Suárez, promoviendo una auténtica desconsideración de sus tesis originales; b. el jesuita español fundaba, igual que Tomás de Aquino, la división de las ciencias especulativas en el acto intelectual de abstracción; c. la diferencia entre ambas concepciones metafísicas no implicaba necesariamente una comprensión epistémica distinta. Ahora bien, contra estas interpretaciones aparece en el siglo XXI otra lectura, donde se acentúa la diferencia decisiva de la filosofía del jesuita del siglo XVI, quien, en letras medievales, escribe y piensa en permanente tensión entre la herencia de la filosofía antigua y medieval por una parte, y una neo-escolástica de aires renacentistas por la otra. De acuerdo con ello, si bien su pensamiento discurre por una tradición aristotelizante del saber, la comprensión de las ciencias se asienta, a diferencia de Tomás de Aquino, en clave objetiva. Sobre esta premisa, el siguiente estudio abordará con exclusividad la originalidad epistémica de Suárez según la división de las ciencias especulativas y reales, mostrando el orden, el principio y las relaciones entre las ciencias física, matemática y metafísica.
\end{abstract}

PALABRAS CLAVE: FRANCISCO SUÁREZ, FÍSICA, MATEMÁTICA, METAFÍSICA, ABSTRACCIÓN FORMAL.

Division, object and abstraction of speculative sciences according to Francisco Suárez

\begin{abstract}
During the last decades of the twentieth century, Francisco Suárez's philosophy was part of a scholastic movement commonly referred to as Thomistic School. The
\end{abstract}


approach perspective, as it is commonly known, placed the speculative position of Suarez closer to the one of the Dominican St. Thomas Aquinas, according to the following nuances: a. in the epistemic sphere, the figure of St. Thomas widely overshadowed that of Suarez, which promoted an authentic disregard of his original thesis; b. like Thomas Aquinas, the Spanish Jesuit founded the division of speculative sciences in the intellectual act of abstraction; $c$. the difference between both metaphysical ideas did not necessarily imply a different epistemic understanding. However, in the $21^{\text {st }}$ century, there appears another text which emphasizes the crucial difference of the Jesuit's philosophy of the $16^{\text {th }}$ century, who, in medieval letters, writes and thinks in permanent tension between the inheritance of the ancient and medieval philosophy on the one hand, and a neo-scholasticism with Renaissance airs on the other. Therefore, although his thinking goes through an Aristotelian tradition of knowledge, the understanding of science is based on an objective base, unlike Thomas Aquinas. Based on this premise, the following research work will exclusively deal with the epistemic originality of Suarez, following the division of speculative and real sciences, and in this way, which shows the order, the principle, and the existing relations between the physical, mathematical and metaphysical sciences.

KEYWORDS: FRANCISCO SUÁREZ, PHYSICS, MATHEMATICS, METAPHYSICS, FORMAL ABSTRACTION.

\section{Consideraciones preliminares}

Suárez ${ }^{1}$ entiende la totalidad de la ciencia humana en términos de orden y jerarquía de conocimientos, donde el obiectum formale indica, en cada caso, el tema central sobre el que versan las ciencias. ${ }^{2}$ Tal afirmación esconde un giro especulativo fundamental entre la escolástica de la baja Edad Media de los siglos XIII-XV y los aires de una nueva impronta científica que adelantan ya en el siglo XVI la Edad Moderna. ${ }^{3}$ En su conjunto, tales concepciones comparten un horizonte de características platónicoaristotélicas cuyos matices parecía conocer bien Francisco Suárez. ${ }^{4}$ Las metafísicas de Alberto Magno, Tomás de Aquino, Capreolo, Ferrariense y Cayetano, como así también aquellas de Agustín de Hipona, Buenaventura y Duns Scoto, figuran entre las páginas que componen las Disputationes Metaphysicae, editadas en la Universidad

1 El siguiente estudio está basado en la traducción de Francisco Suárez, Disputaciones Metafísicas (DM) de Rábade Romeo et. al., 1960. Sin embargo cabe señalar que he tomado cierta distancia respecto de algunos giros gramaticales y términos específicos en razón de una mejor intelección de la lengua latina. Así, v.g., cuando el latín dice intellectus, he preferido traducir "intelecto", y no aquellos términos de "razón" o "entendimiento" según figuran en la traducción supra. También se ha enmendado la versión castellana cuando aparecían ciertas lagunas en comparación con la completa versión latina.

2 Courtine, 2015: 12-41. Este capítulo, dedicado al tratamiento escolástico de la metafísica como ciencia, se detiene en la transformación de la noción de subiectum en obiectum. Sobre la base de numerosos textos que recorren principalmente las posiciones de Avicena, Tomás de Aquino y Duns Scoto, se busca mostrar el giro que comienza en la teología como sacra doctrina hasta su configuración como metafísica en un horizonte cada vez más racionalista. Mientras que la noción de subiectum designa la unidad de una ciencia per se, la noción de obiectum, en cambio, siempre enmarcada en un contexto noético, expresa la relación de la ciencia según la expresión quoad nos. Allí, en su configuración, interviene activamente la inteligencia. A su vez la transformación señalada, añadimos, opera en la totalidad de las ciencias especulativas y reales, lógicas y prácticas.

3 Prieto López, 2013: 4-11. Véase aquí la lenta gestación de posiciones próximas al racionalismo moderno donde la presencia de Suárez en las universidades españolas, francesas, alemanas y holandesas, es decisiva. Nótese también su influencia en numerosos filósofos de la modernidad, tales como Descartes, Leibniz, Wolff y Kant entre otros. Cf. León Florido, 2011: 22-24; 34-49.

4 Después de la condena parisina del año de 1277 por el obispo Esteban Tempier, el aristotelismo en parte se mantiene, y en parte se supera mediante discusión de sus tesis físicas, lógicas y metafísicas. El s. XIV produce un cambio de enfoque en la realidad, cuya manifestación más evidente aparece en los modos de comprensión de las ciencias especulativas. Según este viraje, la revolución científica en tales ámbitos pone de manifiesto el enfrentamiento entre los antiqui y los moderni. Tomás de Aquino quedó entre los antiqui y Duns Scoto entre los moderni, quien, junto con Ockham y Buridan, fueron gestando un nueva intelección de lo real cuyas consecuencias en teología y filosofía alcanzaron tanto a Cayetano como a Suárez. Véanse Gilson y Langan, 1963: 3-7 (la quiebra del aristotelismo); Fabro, 1965: 557-574 (neotomismo y neoescolástica); Verdú Berganza, 1993; León Florido, 2010: 233-289 (cap. 6: la condena de los moderni y la revolución científica medieval); León Florido, 2000 (texto que presenta una aplicación de la estructura de pensamiento aristotélica hasta el s. XVII); Lohr, 1996: 13: "la base fundamental de la reinterpretación cristiana que llevó a cabo Suárez del pensamiento de Aristóteles reside en la relación entre la realidad finita y el infinito poder creador de Dios". 
de Salamanca en el año de $1597.5^{5}$ Sin embargo, la influencia más evidente en su pensamiento proviene de Aristóteles y de la tradición aristotelizante medieval cuyo primer epicentro lo constituye Tomás de Aquino. ${ }^{6}$ De acuerdo con ello, la doctrina epistémica suareciana discurriría sobre la base de las siguientes líneas de pensamiento: 1. Aristóteles, y el aristotelismo medieval y renacentista, 2. Tomás de Aquino y el tomismo medieval de la primera escolástica, 3. la naciente segunda escolástica dominica, entre quienes deslumbra la figura del cardenal Tomás de Vio Cayetano.?

Las filosofías especulativas propias de este arco temporal que se extiende hasta el siglo XVII muestran la configuración de una ciencia de cuño aristotélico que hallaría fundamento en la operación intelectual de la abstracción. Esta ha sido la lectura mayoritaria propia del tomismo científico del siglo XX donde, al menos en lo que respecta a los objetos de las ciencias especulativas, se destacaba que las posiciones científicas de Tomás de Aquino, Tomás de Vio Cayetano y Francisco Suárez presentaban fuertes similitudes. ${ }^{8}$

A su vez, investigaciones en curso desarrolladas en el devenir del siglo XXI han logrado desarticular aquella interpretación que unía tales insignes personalidades, y poner de manifiesto la profunda originalidad y diferencia de los dos primeros. ${ }^{9}$ Sin embargo, también es cierto que la autoridad de Tomás de Aquino sobre Suárez parece mostrarse decisiva. En efecto, siendo citado 1008 veces en las $D M$ se sigue la necesidad de esclarecer, aunque sea brevemente, la posición epistémica del Aquinate.

Para el dominico del siglo XIII la división de las ciencias especulativas es abordada desde la noción de subiectum, genus-subiectum o subiectum scientiae, y la doctrina de la abstracción colabora en la distinción de aquellas ciencias. Lo abstracto, característica constitutiva de la composición y complejidad de la naturaleza, señala, en primera instancia, una característica interna a la noción de ente que guarda correlación, en segunda instancia, con la operación intelectual abstractiva. No hay aquí objetos de ciencias en sentido estricto, sino un subiectum scientiae que oficia de principio y fin de la misma ciencia, y cuyo devenir del subiectum, sobre la base y despliegue del actus essendi, revelaría la constitución de los principios y temas de un ámbito de la naturaleza, ya sea físico, matemático o metafísico. ${ }^{10}$

Ahora bien, el cambio de epicentro desde el subiectum scientiae al obiectum formale trae consigo modificaciones en la comprensión de la doctrina de la abstracción. Esta última noción no opera de la misma manera ni las ciencias se articulan de idéntico modo en Tomás de Aquino o en Suárez. Por consiguiente, si centramos la atención en el Dr.

5 Pueden consultarse para el contexto histórico de las universidades y el saber en general los siguientes textos: Hale, 2016: 293-336; Parker, 2017: 354-373; Rodríguez-San Pedro de Bezares, 1986; Gómez López, 1996; Alejo Montes, 1990.

6 Prieto López, 2013: 162-163: Tomás de Aquino es el segundo autor más citado en las DM con un total de 1008 veces. En primer lugar Aristóteles con un total de 1.735 veces, y en tercer lugar Duns Scoto con 363 veces. Asimismo conviene señalar que si bien Aristóteles encabeza la lista de autoridades, la comprensión de los textos aristotélicos está completamente mediada por la Escolástica Medieval. De allí que las diferencias más sutiles entre las distintas doctrinas científicas en la línea de pensamiento aristotelizante aparezcan por contraposición con aquella mantenida por Suárez, máxime cuando Tomás de Aquino se posiciona como la autoridad más citada.

7 El lenguaje metafísico de Suárez, de marcada influencia escotista, también se hace presente en Tomás de Vio Cayetano. Sobre este respecto, cf. Muñoz, 2016. Véanse especialmente los capítulos III y IV acerca de la ciencia y la objetividad como nuevo ámbito cognoscitivo en comparación con aquel de Tomás de Aquino.

8 Véase Mendoza, 2015; Cuccia, 2015, 2017a; 2017b y 2018.

9 Cf. Bonino, 1997, 2000 y 2007; Lorenz, 2003. Sobre Cayetano, cf. n. 7.

10 Cf. Mendoza, 2017. Véanse allí las diferencias con respecto al doble ordo scientiarum concebido por Tomás de Aquino sobre la base del esse como actus essendi. Por un lado existe un orden quoad nos que atañe al sucesivo descubrimiento de las ciencias especulativas por parte de la persona: $1^{\circ}$ ) el genus-subiectum de la física o ens mobile; $2^{\circ}$ ) el genus-subiectum de la matemática o ens quantum; $3^{\circ}$ ) el genus-subiectum de la metafísica o ens en cuanto ens. Por el otro, el orden se altera según una consideración per se: $1^{\circ}$ ) el genus-subiectum de la metafísica, $2^{\circ}$ ) el genus-subiectum de la física, $3^{\circ}$ ) el genus-subiectum de la matemática. Y en ambos órdenes interviene la doctrina de la abstracción en cuanto es, en primera instancia, constitutiva de los estratos de la naturaleza de los entes. 
Eximio, parece operarse un movimiento traslaticio desde el monje dominico donde la razón se convertiría ahora en un principio activo que colaboraría e intervendría en la definición de los entes en general, modificando de suyo la doctrina de las ciencias en su totalidad. Aquí, a diferencia del Aquinate y de acuerdo con el giro especulativo señalado, el ámbito de las ciencias es objetivo. Y esta es la posición epistémica que sostiene Suárez en sus DM.

Asimismo las investigaciones contemporáneas no habrían profundizado en demasía la concepción científica del jesuita español sostenida en sus $D M$. De allí el interés por mostrar en detalle una introducción al mapa de ciencias especulativas concebido por el Dr. Eximio, quien tan decisiva influencia ejerció en la Modernidad filosófica.

\section{Criterios, finalidad y división de las ciencias especulativas}

El tratado primero de las $D M$ puede ser considerado un texto netamente epistémico. Allí el Dr. Eximio presenta, con ocasión de aclarar el objeto de la metafísica, numerosas disquisiciones acerca de las ciencias. Expone en los primeros cinco capítulos los nombres y las razones de cada uno de tales saberes para finalmente detenerse en las tareas y las vinculaciones entre aquellas ciencias que reciben la denominación de especulativas, sin que ello signifique la ausencia necesaria de los demás ámbitos de conocimiento, tales como la lógica, ${ }^{11}$ las ciencias prácticas ${ }^{12}$ y las artes.

De acuerdo con ello recibe el nombre de ciencia especulativa el ámbito de saber que se detiene en el "conocimiento de la verdad"13 en cuanto "lo común a toda ella es la filosofía de la naturaleza". ${ }^{14}$ Suárez también define lo "especulativo" como "contemplación de la verdad" cuya precisa concreción se da en el juicio que traduce con "fuerza infalible o rectitud" el movimiento de la razón. ${ }^{15}$ De modo que el conocimiento científico especulativo atañe a la razón, como cierta afección del espíritu, que la perfecciona al modo de un hábito mental. ${ }^{16}$ Este hábito emerge de la relación entre el raciocinio especulativo y la naturaleza, en cuanto ella se presenta pasible de conocimiento verdadero según las actividades de la razón: indagación, descubrimiento y contemplación. De donde, al ser el fin de la mente especulativa la contemplación de la verdad en lo natural, la filosofía de la naturaleza, que se dice a la vez ciencia especulativa, consiste en una permanente actividad de la razón sobre la naturaleza en su totalidad.

La ciencia especulativa no es propiamente una ciencia según la especie o el género. Simplemente una denominación supra genérica que contiene bajo sí tres géneros de ciencias que comparten el fin general anteriormente señalado. Una de tales ciencias es la metafísica, ${ }^{17}$ y en ella se considera superlativamente el fin último de todas las demás, ya sean especulativas o prácticas, pues la metafísica "muestra el fin al que se

\footnotetext{
11 En derredor de la diferencia entre lógica y metafísica, cf. Suárez, DM I, IV, 29-33.

12 DM I, IV, 34: “Alia denique consideratio scientiae potest dici moralis, quatenus usus vel excercitium scientiae potest esse laude vel reprehensione dignum; quod munus ad moralem philosophiam vel prudentiam spectat".

13 DM I, V, 45: "At vero dirigere scientias sub priori ratione [ciencia especulativa], scilicet, ad veri cognitionem".

${ }_{14} D M$ I, IV, 3: "communia sunt omnibus speculativis scientiis, et maxime naturali philosophiae, et ita ex dictis rationibus recte infert Aristoteles hanc scientiam speculativam esse, et non practicam, quia proxime non ordinatur ad opus, sed sistit in veritatis cognitione, quod (ut dixi) commune est universae naturali philosophiae".

15 DM I, V, 45: "Oportet ergo in scientiis duo distinguere, scilicet, veritatis contemplationem seu iudicium et infallibilem vim seu rectitudinem attingendi illam...".

16 DM I, IV, 34: “considerationem scientiae, prout est quaedam spiritualis qualitas mentis et habitus".

17 DM I, III, 1: "Deinde constat ex dictis, hanc scientiam [sc. metaphysicam] esse essentialiter distinctam a reliquis scientiis speculativis ac realibus, ut sunt philosophia et mathematica".
} 
han de dirigir todas las cosas y muestra que ese es el fin último de todas las cosas". ${ }^{18}$ Este fin, que es la beatitud natural del hombre, ${ }^{19}$ engloba todo arte y todo conocimiento, identificándose, en un primer momento, con la denominación de ciencia especulativa, ${ }^{20}$ y seguidamente, con los nombres de ciencias prácticas y artes. De modo que el fin último de todo conocimiento, para producir en la caso del arte, para actuar en el caso de las ciencias prácticas, o en sí mismo según los saberes especulativos, es la felicidad humana; y esa felicidad es, en definitiva, la contemplación de la verdad. De esta manera la ciencia metafísica dirige todo otro saber, ${ }^{21}$ y el ingenio humano no se reduce a la metafísica. Por el contrario, solo se colma según la consideración de todas las ciencias.

... non posse dari unam scientiam humanam, et propriis actibus humani ingenii acquisitam quae universaliter illud perficiat quoad omnia scibilia secundum omnes rationes eorum, alioqui non solum illae scientiae speculativae philosophia, mathematica et metaphysica, sed etiam morales et rationales, ac denique omnes in unam coalescerent, quae esset adaequata perfectio intellectus humani (DM I, II, 24).

... no puede darse una única ciencia humana adquirida con los propios actos del ingenio humano, que le perfeccione universalmente con relación a todos los objetos cognoscibles y bajo todos los aspectos de estos, pues de lo contrario, no sólo aquellas tres ciencias especulativas, filosofía, matemáticas y metafísica, sino también las morales y racionales, y todas las demás, finalmente se reunirían en una sola, que vendría a ser la perfección adecuada del intelecto humano (DM I, II, 24).

La idea vuelve a repetirse: "es incomprensible que por sí mismas todas ellas [sc. las ciencias] sean verdadera y propiamente una única ciencia humana". ${ }^{22}$ En cualquiera de sus dos versiones, el criterio general que indica la noción de ciencia, y específicamente las especulativas, es el mismo. Ni la metafísica, ni ninguna otra ostentan el honor de ser una única ciencia que colme el ingenio humano. Esto es sencillamente imposible. Empero, no hay obstáculo para que haya alguna de ellas que se comporte como regente de todas las otras, según quedó manifestado anteriormente.

Ahora bien, la imposibilidad de reducción a una implica de suyo la existencia de múltiples ciencias. De modo que, antes de la mención específica de cada uno de los distintos saberes, aparecen las regiones científicas con sus fines propios. Así las especulativas, las racionales y las morales. En relación con las primeras, Suárez explícitamente menciona tres: filosofía, matemática y metafísica. Ellas configuran la totalidad de ciencias especulativas y comparten un fin general: la contemplación de la verdad. Su realización, en cambio, varía en cada caso. Allí nos dice: "el filósofo rara vez se aparta del conocimiento sensorial. En cambio el metafísico procede por medio de principios universalísimos y en sumo grado abstractos. Y finalmente el

$18 D M \mathrm{I}, \mathrm{V}, 45$ : "ostendit tamen finem in quem omnia dirigenda, eumque finem ultimum rerum omnium esse ostendit".

${ }_{19} D M \mathrm{I}, \mathrm{V}, 45$ : "Addo denique, si metaphysica consideratur quatenus in perfectissimo actu eius naturalis beatitudo hominis consistit, sic ad illam ut ad finem ordinari non solum alias scientias, sed etiam morales virtutes et prudentiam, nam haec omnia ad felicitatem hominis ordinantur, et actiones omnes ad hunc finem optime referuntur". Nótese el idéntico sentido entre los términos beatitud natural y felicidad.

$20 D M$ I, V, 45: "At vero dirigere scientias sub priori ratione, scilicet, ad veri cognitionem, per se primo ac maxime pertinet ad metaphysicam, a qua quodammodo accipiunt principia, et terminorum cognitionem, et obiecta, seu quidditates suorum obiectorum".

$21 D M$ I, V, 45: "Atque hac etiam ratione concludit Aristoteles scientiam hanc omnibus imperare, quia contemplatur summum bonum et ultimum finem simpliciter; sicut enim in artibus, quae ad aliquem finem subordinantur, illa architectonica est, aliisque imperat, quae supremum finem in illo ordine considerat, ita metaphysica, quae absolute contemplatur ultimum finem scientiarum, artiumque omnium, totiusque humanae vitae, dicitur imperare omnibus, esseque omnium princeps, non quia proprie et practice imperet, sed quasi virtute et eminenter".

22 DM I, II, 11: "Addo deinde (quidquid sit de illis tribus scientiis, philosophia, mathematica et metaphysica, quo modo unaquaeque earum per se una sit, quod paulo post breviter attingemus), per sese incredibile esse eas omnes vere ac proprie unicam scientiam humanam esse". 
matemático toma un camino intermedio". ${ }^{23}$ Luego, la verdad en el ámbito de la naturaleza perceptible por los sentidos, la verdad en un ámbito intermedio, y la verdad en una naturaleza en sumo grado abstracta.

Tal delimitación tripartita de lo natural es imposible sin el concurso de la razón humana. En efecto, si la naturaleza se dice pasible de ser indagada por tres ciencias, es porque en sí misma presenta una composición sustancial compatible. Asimismo, dicha relación perfecciona el ingenio humano del siguiente modo: "la filosofía natural [o ciencia física o filosofía] parece que perfecciona el intelecto en cuanto se vale de los sentidos y se ocupa de las cosas sensibles". ${ }^{24}$ En cambio "las ciencias matemáticas parecen perfeccionarlo en cuanto en cierto modo abstraen de la experiencia de los sentidos externos, aunque con dependencia de la imaginación o de la fantasía". ${ }^{25}$ Finalmente la metafísica, pues "ilustra el intelecto según sí mismo, al abstraer de los sentidos y de la fantasía que tienen asidero en el cuerpo, y contempla solo las cosas espirituales y divinas y todas las razones y principios comunes de [todas] las cosas, y los atributos generales de los entes que ninguna ciencia inferior considera". ${ }^{26}$

De las tres descripciones supra se colige lo siguiente: 1. no hay aquí un orden de ciencias perse; únicamente se bosqueja un orden quoad nos; 2. este orden es ascendente y procede de lo más externo a lo más interno. En primer lugar la ciencia física, en razón de la correlación entre los sentidos externos y las cosas sensibles. Seguidamente, la matemática, en cuanto vincula la imaginación -que se diferencia de los sentidos externos sin prescindir absolutamente de ellos, pues dice Suárez "en cierto modo"con la naturaleza sensible. De allí que lo común a ambas ciencias sean las sustancias sensibles y materiales. Y finalmente la ciencia metafísica, al abstraer enteramente de las cosas sensibles, y así considerar las siguientes tareas: ${ }^{27}$ a. las cosas espirituales y divinas: Dios y los ángeles según su naturaleza y operación; b. todas las razones y principios comunes de [todas] las cosas. Respecto de lo primero: la noción de sustancia y accidente en cuanto tal, sin que existan en la materia, y así la noción de ente increado y creado, de sustancia finita e infinita y los accidentes absolutos de la cualidad o la acción. ${ }^{28} \mathrm{Y}$ respecto de lo segundo: "b.1. todo ente es y no es; b.2. es imposible afirmar y negar algo de lo mismo, etc.",29 c. atributos generales de todos los entes: los conceptos trascendentales unum, verum, bonum. ${ }^{30}$

23 DM I, II, 11: "nam philosophus vix recedit a sensu; metaphysicus vero procedit per principia universalissima et maxime abstracta; mathematicus vero medio quodam modo procedit".

${ }_{24}$ DM I, IV, 4: "Philosophia igitur naturalis videtur intellectum perficere, prout utitur sensibus, et circa sensibilia versatur". 25 DM I, IV, 4: "Mathematicae vero scientiae illum videntur perficere, prout aliquo modo abstrahit ab externorum sensuum experimento cum dependentia tamen ab imaginatione, seu phantasia".

26 DM I, IV, 4: "Haec autem doctrina intellectum illustrat secundum se, abstrahens, quantum in corpore fieri potest, a sensibus et phantasia, et res spirituales ac divinas contemplans, rationesque ac principia omnibus rebus communia, et generalia attributa entium, quae a nulla inferiori scientia considerantur".

27 DM, I, IV, 3: "proprius finis huius scientiae declaretur, subintelligere oportet eam esse propter cognitionem earum veritatum quae de ipso ente ut tale est, et de rebus quae secundum esse abstrahunt a materia, demonstrari possunt. Atque ita proprius finis eius est declarare naturam, proprietates et causas entis in quantum ens, et partium eius, quatenus secundum esse a materia abstrahunt".

28 DM I, II, 15: "Atque hinc constat (ne eadem repetamus) idem dicendum esse de omnibus rationibus communibus quae sub ente, substantia et accidente ita abstrahi possunt ut sint in rebus sine materia; huiusmodi sunt ratio entis creati vel increati, substantiae finitae aut infinitae, et similiter accidentis absoluti vel respectivi, qualitatis, actionis, operationis aut dependentiae et similium".

29 DM I, IV, 21: "Quodlibet est, vel non est, et: Impossibile est aliquid de eodem affirmare et negare".

$30 D M$ I, I, 28: "nam revera ens habet suas proprietates, si non re, saltem ratione distinctas, ut sunt unum, verum, bonum, quod ostendemus statim disputatione tertia ubi declarabimus an ens includatur intrinsece et per se in huiusmodi proprietatibus". 


\title{
3. La abstracción formal como principio de distinción
}

El apartado anterior había insinuado una doble división de la naturaleza. Una, las sustancias sensibles, donde aparecen las ciencias físicas y matemáticas. Otra, las sustancias inmateriales, cuya consideración compete a la metafísica. Ahora bien, en orden a comprender por qué los estratos naturales son dos y las ciencias son tres, debemos, según Suárez, atender a la configuración de la ciencia según su esquema triádico fundamental: abstracción formal, objeto formal y método. ${ }^{31}$

\begin{abstract}
Idem repetit lib. VI, a principio, ubi tres speculativas scientias distinguit, philosophiam, mathematicam et naturalem theologiam, quas distinguit tum ex abstractione obiectorum, tum etiam consequenter ex rebus de quibus tractant, tum denique ex modo quo procedunt et demonstrant, ut infra declarabimus. Atque hanc divisionem scientiarum speculativarum secuti sunt omnes Aristotelis interpretes, et fere omnes philosophi (DM I, II, 7).

Lo mismo repite [Aristóteles] en el libro VI [de la Metafísica], desde el principio, donde distingue tres ciencias especulativas, la filosofía, la matemática y la teología natural, y las distingue, ya por la abstracción de sus objetos, ya también consiguientemente por las cosas de que tratan, ya finalmente por el modo con que proceden y demuestran, como luego diremos. Y esta división de las ciencias especulativas ha sido seguida por todos los intérpretes de Aristóteles y por todos los filósofos (DM I, II, 7).
\end{abstract}

La primera distinción corresponde a la llevada a cabo por la abstracción de sus objetos. La segunda por las cosas que tratan. La primera y la segunda se ordenan como principio y fin, pues por medio de la abstracción se configuran los objetos sobre la naturaleza, cuyos objetos llevan consigo el esclarecimiento de sus tareas. Finalmente el método, que es consecuencia de los objetos de cada ciencia, se origina también desde el principio, ya que "la diferencia entre estos tres métodos se origina en la tan conocida triple abstracción de la materia". ${ }^{2}$

Las ciencias físicas y matemáticas arraigan su primera distinción en el acto abstractivo del intelecto humano. En cambio la metafísica o teología natural depende de la consideración de la abstracción según el ser y la razón. En los tres casos la abstracción de sus objetos, que es la consideración de los entes tomados en universal, configura un marco propicio donde se manifiestan los objetos formales propios de cada de una de las ciencias, puesto que tales objetos muestran "las quididades de los entes que están contenidas bajo su forma propia de abstracción, o en cuanto necesariamente se encuentran unidas con ella". ${ }^{33}$

\footnotetext{
Illae ergo tres scientiae in aliqua abstractione conveniunt: nam omnes considerant de rebus in universali; differunt tamen in abstractione quasi formali et praecisiva a materia, nam philosophia quamvis abstrahat a singularibus, non tamen a materia sensibili, id est, subiecta accidentibus sensibilibus, sed ea potius utitur in suo ratiocinandi modo. Mathematica vero abstrahit quidem secundum rationem a materia sensibili, non autem ab intelligibili, quia quantitas, quantumvis abstrahatur, non potest concipi nisi ut res corporea et materialis. Metaphysica vero dicitur abstrahere a materia sensibili et
}

31 DM I, II, 11: "cum ergo mathematica distincta a philosophia sit omnium consensu, ob diversam abstractionem modumque procedendi omnino diversum, idem maiori ratione censendum est de metaphysica respectu illarum". El siguiente estudio únicamente aborda los dos primeros tópicos, postergando para fututos trabajos la cuestión del método científico en Suárez.

32 DM I, II, 11: "qui modi procedendi oriuntur ex illa vulgati triplici abstractione a materia".

33 DM I, II, 13: "haec scientia [sc. metaphysica] non considerat omnes proprias rationes seu quidditates entium in particulari, seu ut talia sunt, sed solum eas quae sub propria eius abstractione continentur, vel quatenus sunt cum illa necessario coniunctae". Y esto último acontece en cada una de las ciencias especulativas. 
intelligibili, et non solum secundum rationem, sed etiam secundum esse, quia rationes entis quas considerat, in re ipsa inveniuntur sine materia; et ideo in proprio et obiectivo conceptu suo per se non includit materiam (DM I, II, 13).

Aquellas tres ciencias convienen, pues, en cierta abstracción, pues todas dirigen su consideración a las cosas tomadas en universal. Sin embargo difieren en la abstracción cuasi-formal y precisiva de la materia, pues la filosofía, aunque hace abstracción de lo singular, no hace así con la materia sensible, es decir, la que está sujeta a los accidentes sensibles, sino que más bien se vale de ella en su modo de raciocinar. En cambio la matemática abstrae según la razón de la materia sensible, aunque no de la inteligible, porque la cantidad, por mucho que se abstraiga, no puede concebirse más que como una cosa corpórea y material. Por su parte la metafísica se dice que abstrae de la materia sensible e inteligible, no sólo según la razón, sino según el ser, porque las razones de ente que considera se hallan en la cosas sin materia y, por tanto, en su concepto propio y objetivo de por sí no incluye la materia (DM I, II, 13).

Si la naturaleza sensible está conformada por la totalidad de sustancias materiales y singulares, se sigue que la abstracción, para considerar lo que en ellas hay de universal, es un acto de la inteligencia. De allí que la abstracción intelectual sea formal o cuasi-formal y precisiva, porque atiende al ámbito de la física, donde se considera la forma, que es el principio por el cual se manifiesta lo que es la sustancia, en universal. La ciencia física prescinde de lo singular, no de la materia sensible a la que le quedan sujetos los accidentes sensibles. De allí la tarea y definición de la filosofía natural, ya que la descripción de las sustancias físicas se asienta sobre el movimiento discursivo de la razón humana que previamente ha abstraído el objeto formal de sus consideraciones. Por esta razón, el Dr. Eximio afirma que la física tiene como objeto científico la forma universal -de ahí su razón de objeto- de los entes sensibles o materiales, cuya singularidad queda apartada o separada, y siendo, sin embargo, su materialidad sensible parte de su definición. Abstracción formal en física significa considerar simultáneamente la forma y desconsiderar la singularidad de la materia. Y así la separación de la materia hace que la forma aparezca según su universalidad, y no concretada en la materia singularizada. Por esta razón, la abstracción de la forma configura la abstracción formal, y lo abstraído, su universal. Por ello el universal de la abstracción intelectual recibe el nombre de objeto formal.

Así como la ciencia física separa la singularidad de la materia sensible, así también la matemática separa esta última. Hay un proceso racional y conceptual de remoción material. Primero la física, donde el objeto formal incluye la materia sensible ausente de singularidad, y luego la matemática, donde se remueve o abstrae la materia sensible, quedando únicamente presente para la razón la materia inteligible. De donde la forma sustancial de los entes materiales, abstracta por la razón de materia sensible, permanece en y se muestra a través de la materia inteligible. Y esta es la explicación del accidente de cantidad dado siempre en las sustancias materiales. Finalmente, la abstracción según la razón de la forma respecto de la singularidad, constituye con propiedad el primer grado de abstracción, que es aquel de la ciencia física. De este modo la filosofía natural se ubica en el primer grado de abstracción formal, y aparece como la primera ciencia quoad nos en el orden de la ciencias especulativas. De idéntica manera sucede con la ciencia matemática, puesto que al tener como objeto formal la cantidad, queda ubicada en el segundo grado de abstracción formal, al mostrarse ella misma como la segunda ciencia especulativa quoad nos.

Física y matemática atañen a la consideración de los entes sensibles y materiales. El caso de la metafísica es diferente. Aquí los entes de suyo están ausentes de materia sensible e inteligible. De donde la noción de abstracción en este contexto señala la remisión total de ambos modos materiales y la sola presencia del ente espiritual. 
Hay aquí negación y distanciamiento de los entes sensibles, pues el intelecto separa y desconsidera la totalidad de tales entes. Esta es la diferencia entre las tres abstracciones. La primera y la segunda apuntan al ente material. En cambio la tercera, al ente espiritual. Ahora bien, los entes espirituales no se alcanzan para el hombre por la sola abstracción del intelecto respecto de los entes materiales. Ello es únicamente el aspecto negativo, donde se afirma que las ciencias físicas y matemáticas carecen de competencia para tratar acerca de los entes espirituales. En cambio el aspecto positivo está señalado por la abstracción según el ser. En esta ocasión lo que allí se indica es la necesidad de ser de los entes espirituales, asegurando la existencia de tales entes y eliminando la duda de que ellos sean una mera proyección metafísica irreal. Por lo tanto, la abstracción metafísica, a diferencia de las anteriores, si afirma positivamente la necesidad de la existencia de tales entes, y constituye de suyo el tercer grado de abstracción en una consideración especulativa quoad nos.

\footnotetext{
De qua triplici abstractione et partitione. harum trium scientiarum per has abstractiones ex professo disseritur in libris Posteriorum. Nunc nobis sufficiat hactenus non esse inventam aptiorem rationem distinguendi has scientias, et aliunde hanc videri satis convenientem; nam, cum hae scientiae sint de rebus ipsis, sintque maxime speculativae, ideoque abstractione utantur, ut constituant obiectum scibile de quo possint demonstrationes fieri, recte ex diverso modo abstractionis intelligitur variari obiectum scibile ut sic; et ideo solet dici haec abstractio, quatenus in ipso obiecto fundamentum habet, ratio formalis sub qua talis obiecti in ratione scibilis (DM I, II, 13).
}

Sobre esta triple abstracción y sobre la división de estas tres ciencias tratan exprofesso los Analíticos Segundos. Por ahora bástenos saber a nosotros que no ha sido hallada una manera más apta de distinguir estas ciencias, y que, por otra parte, parece bastante idónea, pues versando estas ciencias sobre las cosas mismas, y siendo especulativas en grado máximo y que tienen que usar, por tanto, de la abstracción para constituir el objeto cognoscible, término de sus demostraciones, se sigue lógicamente que según sea el diverso modo de abstracción, cambiará el objeto cognoscible en cuanto tal. Y por ello suele decirse que esta abstracción, en cuanto tiene fundamento en el objeto mismo, es la razón formal sub qua de tal objeto en cuanto cognoscible (DM I, II, 13).

La confirmación del Dr. Eximio acerca de la idoneidad de la doctrina de la abstracción para distinguir las ciencias especulativas ratifica la posición previa que tiene esta operación intelectual respecto de la constitución de los objetos especulativos. El texto afirma que tales ciencias versan acerca de "las cosas mismas", siendo ellas la naturaleza según sus diferentes estratos: los entes físicos y metafísicos. Sobre los primeros, las ciencias físicas y matemáticas; sobre los últimos, la ciencia metafísica. Esta es la naturaleza en su totalidad que es aprehendida vía abstracción por la mente humana. De allí que sean "especulativos en grado máximo", pues el límite de la razón especulativa está en el conocimiento de los entes naturales, cuya verdad, asequible mediante su aprehensión como objeto, permite la profundización en cada género de ciencia, y la consiguiente división interna de la física y de la matemática. Sobre esto último anota Suárez: "suele originarse esta dependencia de una ciencia respecto de otra por la subordinación de sus objetos, pues como el ser de la ciencia consiste en la relación al objeto, así también los principios están proporcionados a este". 34

Ahora bien, la abstracción en general señala la operación intelectual por la que se aprehende el universal físico, matemático o metafísico. Por tal motivo el acto de abstraer es previo al objeto, y también límite constitutivo de los objetos de las ciencias en cuestión. A su vez, el objeto formal de la física, la matemática o la metafísica

$34 D M$ I, V, 49: "Oriri autem solet haec dependentia unius scientiae ab alia ex subordinatione obiectorum; nam, sicut esse scientiae consistit in ordine ad obiectum, ita et principia sunt proportionata illi". 
se dice término de demostración en cuanto los argumentos científicos hacen foco en demostrar que ellos son con propiedad el fin de cada una de estas áreas de saber. Son demostraciones que se dirigen a mostrar los objetos de las ciencias, y no demostraciones que lo tienen por supuesto para señalar deducciones del mismo objeto. De acuerdo con ello, si la abstracción es principio operativo intelectual, "se sigue lógicamente que según sea el diverso modo de abstracción, cambiará el objeto cognoscible en cuanto tal". Debe cambiar primero la abstracción-v.g. de la física a la matemática- y después, a modo de consecuencia, cambiar el objeto. Y ello es así porque al ser humano le es imposible aprehender otro objeto de ciencia con la misma abstracción. En la medida que cambie de abstracción podrá aprehender otro objeto científico.

Abstracción y objeto de ciencia son a la razón humana simultáneos. Solo así puede afirmarse que la abstracción configure el objeto de ciencia y luego que esta operación se funde sobre el objeto mismo. Hay una circularidad cognoscitiva por la cual el objeto no es de una ciencia sino por la abstracción, y la abstracción no es tal sino es por el objeto de alguna de las ciencias especulativas. Ahora bien, solo en virtud de esa circularidad se puede afirmar que el acto abstractivo expresa "la razón formal sub qua de tal objeto en cuanto cognoscible". No es simplemente la razón formal del objeto, sino la razón formal sub qua, o lo que es lo mismo, la operación racional por medio de la cual se aprehende el universal de una de las ciencias especulativas. La expresión sub qua señala la diferencia entre los actos intelectuales abstractivos. Y añade: "en cuanto cognoscible" y no en cuanto conocido, porque aquí se trata de la aprehensión de un posible objeto, que no puede serlo sino en cuanto objeto, forma universal del ente en cuestión, y no en cuanto ente sin más.

\footnotetext{
Item, quia res eo sunt perfectius intelligibiles, quo magis abstrahunt a materia: et similiter cognitio, quo est de obiecto immaterialiori et consequenter abstractiori, eo est certior; et ideo ex diverso gradu abstractionis seu immaterialitatis recte consideratur varietas obiectorum scibilium et scientiarum (DM I, II, 13).
}

Y asimismo, porque las cosas tienen tanta mayor perfección en su inteligibilidad, cuanta mayor es su abstracción de la materia, y de modo parecido, el conocimiento que es del objeto más inmaterial, y consecuentemente, más abstracto, es más cierto. Y por esto, por el diverso grado de abstracción o inmaterialidad rectamente se considera la variedad de los objetos cognoscibles y las diversas ciencias (DM I, II, 13).

La abstracción como operación intelectual no es el único sentido del término. Todo ente, según su composición, es más abstracto o inmaterial, o menos abstracto y material. Este significado tiene como correlato aquel otro de la operación intelectual de abstraer. De allí que los entes sean más inteligibles, o mejor aprehensibles para el intelecto, en la medida que menos materialidad presenten. Y como la materia es doble, se sigue que el objeto de la ciencia es triple. Si atendemos a la materia singularizada, entonces es menos cognoscible para la razón según sí mismo el ente físico, y de allí la necesidad de la abstracción de primer grado. En cambio, si la materia es inteligible, la abstracción formal de su objeto es aquella de la matemática o de segundo grado. Finalmente, si el ente, según su composición, carece de materia en absoluto, su inmaterialidad es total y su inteligibilidad es plena. Por ello los entes metafísicos son máximamente intelectuales y se corresponden con el tercer grado de abstracción formal. En este caso se comprende de mejor modo el doble significado de la noción de abstraer, porque la abstracción según el ser manifiesta de suyo el máximo grado de inmaterialidad de estos entes, siendo su correlato la abstracción según la razón sub qua de tales entes, que es, como se ya se ha afirmado, aquella de tercer grado. 


\section{Objetos formales: límite y relaciones entre las ciencias especulativas}

En virtud de la circularidad entre el acto de abstracción intelectual y la configuración del objeto formal acontece la delimitación de los ámbitos científicos. En efecto, "si no se le asigna el limite por la razón formal del objeto, no hay ningún otro motivo para asignárselo". ${ }^{35}$ Luego, si consideramos que la abstracción es con vistas al objeto formal, auténtico fundamento de cada una de las ciencias especulativas, se comprende entonces que el límite entre tales saberes está dado por la razón formal de objeto.

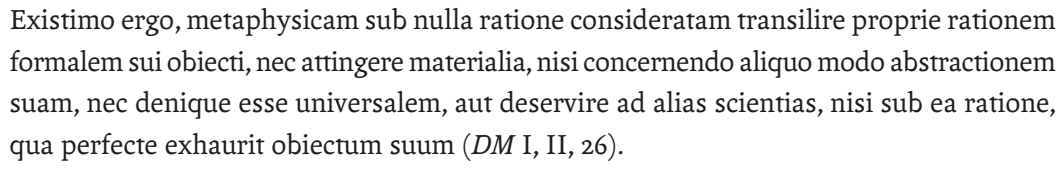

Pienso, por tanto, que la metafísica bajo ninguna razón considerada traspasa propiamente la razón formal de su objeto; ni puede ocuparse de las cosas materiales, sino sólo de las que convienen en su propio grado de abstracción; ni que, finalmente, es universal o sirve a las demás ciencias, sino bajo la razón única en la que agota perfectamente su propio objeto (DM I, II, 26).

Cada ciencia tiene su propia razón formal de objeto que es, a la vez, límite ad intra y ad extra. En relación con lo primero enuncia sus temas propios, mientras que respecto de lo segundo afirma el modo de relación con las demás ciencias. El objeto formal es límite relacional, ya porque una ciencia no se confunde con otra, ya porque pertenecen a distintas abstracciones. La definición de una ciencia, que es por su objeto formal, conlleva tareas ad intra que sugieren dos cuestiones. En primer lugar, el desarrollo de los temas fundamentales de una ciencia, y seguidamente, las divisiones entre las ciencias del mismo género. Ahora bien, en las consideraciones de tales divisiones la primacía la continúa teniendo el objeto formal. De allí que no sean de idéntico modo las relaciones entre las ciencias pertenecientes al mismo género, v.g. desde la física hacia la geología o la biología, que aquellas entre géneros diferentes, tales como metafísica y matemática o física. Por esta razón cada ciencia versa acerca de su propio objeto formal y se extiende hasta donde sea su grado de abstracción.

Las disquisiciones acerca del grado de abstracción y consiguiente objeto formal son válidas para las tres ciencias especulativas sin más. Sin embargo la metafísica, sin deja de cumplir este requisito, presenta una particularidad. Entre las tareas que le son propias está aquella de dirigir las demás ciencias al modo de princesa y señora. A diferencia de las otras ciencias donde las relaciones entre los géneros de la física y la matemática no se interpretan como el gobierno de una sobre la otra, la metafísica, según su límite ad intra, supone principios rectores para sí misma y para las demás áreas del saber. Aquí se pone de manifiesto que el tercer grado de abstracción trae consigo asumir cierta regencia sobre la física y la matemática hasta donde lo permita su objeto formal propio, sin que ello signifique confusión o desacertada intromisión con respecto a aquellas.

Ex hac autem recepta doctrina facile intelligitur et probatur assertio posita, quia scientia non transgreditur limites sui obiecti formalis, seu rationis formalis sub qua sui subiecti; considerat autem quidquid sub illa continetur; ergo haec scientia considerat omnia entia seu rationes entium quae sub praedicta abstractione continentur; et ultra non progreditur, nam caetera ad physicam vel mathematicam spectant (DM I, II, 13).

$35 D M$ I, II, 25: "Sequela vero patet, tum quia si ex ratione formali obiecti non assignatur talis terminus, non est unde assignetur". 
Aceptada esta doctrina, fácilmente se entiende y aprueba la aserción que establecimos antes, que ninguna ciencia sobrepasa los límites de su objeto formal o de la razón formal sub qua de su sujeto, sino que considera todo cuanto en ella se contiene. Luego, esta ciencia [sc. la metafísica] extiende su consideración hasta todos los entes o razones de ser que se contienen en la referida abstracción, y no pasa más allá porque lo que queda es propio del campo de la física o de la matemática (DM I, II, 13).

Si la metafísica no puede avasallar los objetos de las demás ciencias y sin embargo puede pronunciarse sobre ellas, se debe a su grado de abstracción y objeto formal sub qua. ¿Qué dice la metafísica a título de princesa y señora? Suárez afirma: "delimitar los objetos propios de las demás ciencias es oficio propio de la metafísica". ${ }^{36}$

Nam metaphysica non nisi ratione sui obiecti abstractissimi et universalissimi dicitur esse universalis scientia, et principia universalia tradere, ideoque posse alias scientias iuvare [...] sed simul, dum suum obiectum proprium perfecte tractat, consequitur quidquid perfectionis habet super alias scientias, et confert omnem utilitatem quam ad illas praestare potest" (DM I, II, 25).

La metafísica como ciencia que señorea a todas las demás [...] se dice que es ciencia universal y que suministra conceptos universales no por otra razón, sino por causa del grado sumo de abstracción y universalidad de su objeto, gracias al cual puede servir de ayuda a las demás ciencias [...] al mismo tiempo que trata perfectamente de su objeto propio, alcanza cuanto tiene de perfección sobre las demás ciencias y les proporciona toda la utilidad que puede prestarles (DM I, II, 25).

Cada ciencia es universal según sí misma y versa sobre un género científico diferente. La universalidad de sus objetos depende de la abstracción operada en relación con la naturaleza. El objeto formal de la metafísica, que es el concepto de ente en cuanto ente, es útil de suyo a la totalidad de las ciencias físicas y matemáticas, porque no sólo delimita los ámbitos de estas ciencias, sino porque al hacerlo también le presta sus principios, los que, al ser universales, se aplican por extensión a todo el saber especulativo. Así se comprende que, dentro de su propio ámbito, la metafísica sea útil a las demás ciencias, porque en su relación con ellas, y en virtud de su carácter universal, les proporciona junto con la delimitación de sus objetos, una universalidad restringida y unos principios universales directrices. De allí que la regencia de la metafísica sobre la física o la matemática sea cuasi formal, mientras que ellas se comportan al modo de la materia informada, cuyo carácter es cuasi material.

... unaquaeque scientia tradens cognitionem sui obiecti sufficienter reddit facilem intellectum ad distinguendum illud a reliquis, si tamen reliqua cognoscantur, unde duae scientiae possunt sese iuvare et concurrere ad huiusmodi iudicia, maxime si una praebeat veluti formale medium, alia vero quasi ministret materiam, cui medium illud applicatur (DM I, II, 24).

... ya que cada una de las ciencias, al darle el conocimiento de su propio objeto, hace al intelecto suficientemente capaz para distinguir a éste de los restantes, suponiendo que las demás cosas sean conocidas. Por donde se ve que dos ciencias pueden ayudarse y cooperar a tales juicios, sobre todo si una suministra el medio cuasi formal y la otra la cuasi materia a la que se aplica ese medio (DM I, II, 24). 


\title{
5. Las relaciones entre las ciencias: subalternación y división
}

La primera división entre las ciencias especulativas trae consigo una subordinación de saberes. En un orden quoad nos, "esta ciencia [sc. la metafísica] ha exigido siempre, en el orden de nuestro conocimiento, el último lugar para sí [...] pues a causa de ello la llamó transfísica o postfísica". ${ }^{37}$ Sobre este orden ya se había adelantado el lugar de cada ciencia, donde la física ocupa el primero, continúa la matemática y finaliza la metafísica. El argumento que lo confirma dice así:

\begin{abstract}
Et ratio est, quam tetigit D. Thomas, In I Metaph., c. 1, lect. 2, et Avicen., lib. I suae Metaph., c. 3, quia res, quae a materia abstrahunt secundum esse, licet secundum se sint maxime intelligibiles, a nobis tamen non inveniuntur nisi per motum, ut constat ex XII Metaph. Et similiter rationes entis universalissimae et abstractissimae, quamvis secundum se sint notiores, praesertim quoad quaestionem an sint, tamen quid sint, et quas proprietates habeant, difficile a nobis cognoscitur; et saepe incipere oportet a particularibus et sensibilibus, ut ad illas pertingere possimus (DM I, IV, 13).

... porque las cosas que abstraen de la materia según el ser, aunque de por sí sean las más inteligibles, para nosotros no son descubiertas sino mediante el movimiento, según consta en el libro XII de la Metafísica. Y de modo semejante, las razones comunes más universales y abstractas del ente, aunque de por sí sean más conocidas, especialmente en la cuestión de su existencia, con todo nos resulta difícil conocer qué son y qué propiedades tienen, y con frecuencia tenemos que partir de las cosas particulares y sensibles para poder llegar hasta aquellas (DM I, IV, 13).
\end{abstract}

Hay además otro orden pertinente para las ciencias especulativas que pone de manifiesto cierta circularidad de saberes. En este caso, primero aparece la metafísica, en segundo lugar la física y finalmente la matemática. En efecto, al tener esta última ciencia fundamento en la sustancia sensible y material, es necesario primero considerar el objeto formal de la física, que se corresponde con el primer grado de abstracción formal, y luego considerar, mediante el segundo grado de abstracción, el accidente de cantidad, objeto formal de la matemática. A su vez, entre ambos órdenes cabe señalar la primacía de la jerarquía quoad nos puesto que los grados de abstracción formal se corresponden con este orden, mientras que el enunciado como perse quedaría soslayado y, para el tomismo en general, olvidado.

Unde consequenter fatendum est, si ordo doctrinae spectetur secundum se, metaphysicam esse caeteris priorem [...] nam confirmat aliquo modo omnium scientiarum principia. Deinde transcendentales rationes entis declarat, sine quarum cognitione vix potest in aliqua scientia quidpiam exacte tractari (DM I, IV, 13).

Hay que admitir que si el orden de las ciencias se considera en sí mismo, la metafísica es anterior a las restantes ciencias [...] porque de alguna manera confirma los principios de todas las ciencias. Además, declara las propiedades trascendentales del ente, sin cuyo conocimiento apenas se puede tratar cosa alguna con exactitud de cualquier ciencia ( $D M$ I, IV, 13).

La física es ciencia primera quoad nos, y el descubrimiento de sus principios permiten, por un lado, su aplicación sobre áreas físicas más específicas. Por el otro, el descubrimiento de la ciencia metafísica. Mas el razonamiento metafísico aparece con lentitud para el pensar que dirige y corrige los principios de la ciencia física cuya aplicación

37 DM I, IV, 13: "Nihilominus tamen ratione nostri modi cognoscendi, haec scientia postremum locum sibi vindicavit, ut constat ex usu omnium, et ex ipsamet Aristotelis inscriptione; nam propterea hanc scientiam transphysicam seu postphysicam vocavit". 
no está exenta de errores. Solo un ejercicio constante de la razón en clave metafísica ilumina reflexivamente las ciencias inferiores, enmendando, cuando sea el caso, los principios directrices de las demás ciencias ${ }^{38}$ mediante la determinación de sus objetos formales sobre los que actúan las razones y principios comunes. ${ }^{39}$ La metafísica es ciencia última quoad nos y primera per se. Por lo tanto, la única consideración por medio de la cual la persona accede a esta ciencia en cuanto tal proviene de un tratamiento especulativo que comienza en la filosofía natural. Solo un progresivo avance en la misma permitirá el descubrimiento de aquella ciencia máximamente universal que permitirá eventualmente reconsiderar el orden quoad nos y mostrar la regencia absoluta del orden perse, pues

quia haec scientia considerat supremas entium rationes et universalissimas proprietates, et propriam rationem essentiae et esse, et omnes modos distinctionis qui sunt in rebus; at sine horum omnium distincta cognitione non potest perfecta particularium rerum cognitio haberi (DM I, IV, 5).

esta ciencia [sc. la metafísica] considera las razones supremas de los entes y las propiedades más universales, y la razón propia de la esencia y el ser y todos los modos de distinción que hay en las cosas, sin cuyo conocimiento distinto no es posible lograr una ciencia perfecta de las cosas particulares (DM I, IV, 5).

Ahora bien,

Non est enim necesse ut scientia, quae considerat universalem rationem, in particulari descendat ad omnia quae sub tali ratione continentur, sed solum ad ea quae eamdem rationem scibilis, seu eamdem abstractionem participant (DM I, II, 22).

No es necesario que la ciencia que estudia una razón universal descienda en particular a todas las cosas que se contienen bajo la misma, sino sólo a aquellas que participan de la misma razón de cognoscible o del mismo grado de abstracción (DM I, II, 22).

La división de la ciencia física se explica desde el objeto formal del ente natural, mientras que aquella de la matemática por el objeto formal de la cantidad. Ambas se extienden hasta donde corresponde su grado de abstracción. En el primer caso los entes comprendidos en la física tienen en común la consideración del ente natural que es material y sensible. Y así, en y desde el primer grado de abstracción se señalan las características universales del ente natural y las particularidades que presenta en su configuración. Aquí se contempla la extensión y descenso de la física hacia las ciencias físicas particulares según los principios universales de la primera, de los cuales dependen los principios específicos de las ciencias que le quedan subalternadas. ${ }^{40}$ De idéntico modo sucede en las ciencias matemáticas cuando el objeto formal de la cantidad se extiende, en marco del segundo grado de abstracción formal, a las ciencias de la geometría ${ }^{41}$ y la aritmética, cuyas divisiones

$38 D M$ I, V, 51: "Primum enim non pendent omnino aliae scientiae in esse scientiae a metaphysica, quia non pendent in omni evidentia et certitudine suorum principiorum. Habent enim sua principia immediata, et indemonstrabilia ostensive et directe; quod satis est ut possint habere evidentiam eorum immediate ab habitu principiorum, quae sufficit ad generandam scientiam".

$39 D M$ I, IV, 12: "quamvis metaphysica per se non versetur circa res omnes secundum proprias rationes earum, tamen aliquo modo attingit illas, quatenus, scilicet, necesse est ad proprias rationes explicandas, vel divisiones tradendas, et proprios gradus metaphysicos secernendos ab aliis, quae divisiones metaphysicae multum aliis scientiis deserviunt ad sua subiecta praescribenda, et ab aliis secernenda".

$40 D M$ I, V, 47: "supponamus proprie scientiam illam dici subalternatam alteri, quae essentialiter seu necessario ex natura rei ab illa pendet in esse scientiae, ita ut esse scientia non possit nisi scientiae subalternanti coniungatur, et ab illa sumat evidentiam principiorum. Ratio autem huius est, quia scientia subalternata non habet principia per se nota et immediata, sed conclusiones demonstrabiles in superiori scientia; et ideo, sicut omnis scientia ab habitu principiorum essentialiter pendet...". 
son la ciencia de la música y de la perspectiva. ${ }^{42} \mathrm{~A}$ su vez ambos géneros de ciencias especulativas están subordinados esencialmente al ente, que es aquel de la metafísica, pues

omnes enim aliae scientiae saepe utuntur principiis metaphysicae, aut ea supponunt, ut in suis demonstrationibus vel ratiocinationibus progredi possint, unde saepe contingit ex ignoratione metaphysicae in aliis scientiis errari ( $D M \mathrm{I}, \mathrm{IV}, 5)$.

todas las demás ciencias con frecuencia acuden a los principios de la metafísica, o bien los suponen, para poder avanzar en sus propias demostraciones o razonamientos, con lo cual se explica que muchas veces se yerra en las otras ciencias por desconocimiento de la metafísica (DM I, IV, 5).

Y ello se cumple en virtud de los principios máximamente universales de la filosofía primera que iluminan los géneros de la física y la matemática de modo esencial, y no secundum esse. En efecto

Rursus obiecta inferiorum scientiarum non sunt per accidens subordinata enti aut substantiae; sed per se et essentialiter, ut patet in ente naturali, quod est obiectum philosophiae, et de quantitate, quae est obiectum mathematicae (DM I, V, 51).

los objetos de las ciencias inferiores no están subordinados accidentalmente al ente o a la sustancia, sino por sí mismos y esencialmente, como se ve claramente en el ente natural, que es el objeto de la filosofía, y en la cantidad, que es el objeto de las matemáticas ( $D M$ I, V, 51).

La subordinación esencial atañe a la dependencia de las ciencias inferiores respecto de la metafísica, pues "no existe verdadera subalternación más que entre ciencias que son diferentes". ${ }^{43}$ En efecto, siendo que los principios físicos dependen según sí mismos de la ciencia física, y según la metafísica, de la filosofía primera, se sigue necesariamente que la división entre física, matemática y metafísica se ordena según una relación de libre dependencia de una ciencia respecto de otra. Y esta dependencia se manifiesta siempre subalterna de la metafísica cuando se señalan las relaciones de la física y la matemática respecto de la ciencia máximamente universal.

Hay aquí una relación epistémica donde prima la constitución de los entes. Según la composición que presenten, pertenecerán al ámbito especulativo de una u otra ciencia, siendo la matemática próxima a la física al sustentarse en los entes sensibles. No hay en esto vinculación al modo de la existencia actual de los entes. Basta que sus objetos sean posibles. La física y la matemática no llevan consigo la necesidad de existencia del ámbito de los entes que considera. En cambio en la metafísica la identidad entre esencia y ser supone la necesidad de existencia. Veamos ello con mayor precisión:

\footnotetext{
... hanc scientiam ita iuvare alias ad ostendendum earum obiecta esse, vel quid sint, sicut ipsae de obiectis suis hoc supponunt. Scientiae autem, per se loquendo, non supponunt suum obiectum actu existere; hoc enim, ut argumentum factum probat, accidentarium est ad rationem scientiae; illam excipio, quae est de Deo, cuius esse est de quidditate eius: in aliis vero rebus, ad scientiam et demonstrationem non est necessaria existentia earum $(D M I, I V, 10)$.
}

dicitur illi subalternata, non in totum, sed ex parte, seu partiali subordinatione, non totali; quo modo geometria dicitur subalternari naturali philosophiae, quia, licet utatur multis principiis indemonstrabilibus, aliqua tamen habet quae in philosophia demonstrantur".

42 DM I, V, 50: "Ad subalternationem ergo absolutam et totalem, necesse est ut subiectum subalternatae scientiae addat accidentalem differentiam subiecto scientiae subalternantis, ut linea visualis addit lineae, numerus sonorus numero". 
... esta ciencia ayuda a las demás a mostrar que sus objetos son o qué son en el grado en que ellas mismas lo suponen respecto de sus objetos. Porque las ciencias, hablando absolutamente, no suponen que su objeto existe en acto, según ya se ha probado, ya que esto es accidental para la razón de ciencia, exceptuando a la que trata de Dios, cuyo ser es su esencia; pero en las demás cosas, para la ciencia y la demostración no es necesaria su existencia (DM I, IV, 10).

La ciencia física considera el objeto formal del ente sensible y material. Todos los entes que están bajo este ámbito son tratados por dicha ciencia atendiendo a cada una de sus particularidades. De acuerdo con ello,

Unde, sicut philosophia considerans de variis speciebus substantiarum materialium, considerat subinde communem rationem materialis substantiae, et adaequata principia, et proprietates eius; rursusque agens de variis speciebus viventium, considerat communem rationem viventis ut sic, et propria principia, et proprietates eius; ita scientia humana (ut sic dicam) considerans varios gradus et rationes entium, necesse est ut consideret communem rationem entis (DM I, II, 14).

La filosofía considera las varias especies de sustancias materiales, considera al mismo tiempo la razón común de sustancia material y sus propiedades y principios adecuados; y después, al tratar de las diversas especies de vivientes, considera la razón común de viviente en cuanto tal, y sus propios principios y propiedades; así la ciencia humana -por llamarla así- al considerar los varios grados y razones de ente, es menester que considere la razón común de ente ( $D M \mathrm{I}, \mathrm{II}, 14)$.

Nótese la división de las ciencias físicas de acuerdo con las sustancias abióticas por un lado, y los seres vivientes por el otro. La primera de estas ciencias subalternadas podría nominarse geología, al tratar sobre la totalidad de sustancias materiales, sensibles y singulares que de suyo carecen de vida. Y este nombre es con vistas a los entes infra lunares. Características similares tienen los entes celestes, y la ciencia que los considera es la astronomía. A su vez, ambas ciencias anteceden lógica y físicamente a las ciencias biológicas. Aquellas son inferiores y previas. Estas son superiores y posteriores. La ciencia biológica que corona la totalidad de estos estratos -plantas, animales y humanos- es la antropología. 44

Primo, quia scientia de homine, ut homo est, physica est [...] omnis autem cognitio per materiam physica est. Non est ergo dubium quin cognitio animae, quantum ad substantiam eius, et proprietates per se illi convenientes, et modum seu statum existendi vel operandi quem habet in corpore, ad physicum pertineat (DM I, II, 20).

La ciencia que trata del hombre en cuanto tal es la física [...] ahora bien, todo conocimiento ejercido por medio de la materia es físico. No hay duda, pues, de que el conocimiento del alma, en cuanto a su sustancia y a las propiedades que le convienen por sí, y al modo o estado de existencia u operación que tiene en el cuerpo, pertenece a la física ( $D M I, I I, 20)$.

\section{Consideraciones finales}

El particular giro especulativo acaecido en Francisco Suárez en derredor de la interpretación objetiva de los órdenes de las ciencias, sus divisiones y relaciones de subordinación, muestra una nueva lectura de la tradición medieval que había excogitado

44 DM I, V, 49: "Oportet ergo ut haec subalternatio in obiectis fundetur, nimirum in eo quod obiectum unius est idem cum obiecto alterius, adiuncta aliqua differentia accidentali, quae in esse entis sit per accidens, in esse autem scibilis sit aliquo modo per se, et constituat speciale obiectum scibile...". 
el pensamiento de Aristóteles. Ciertamente el Dr. Eximio se inscribe entre aquellos teólogos que suscribieron la línea de desarrollo científico de raíz aristotélica, y más precisamente, de acuerdo con aquella hermenéutica realizada por Tomás de Aquino. Empero, esta matriz de pensamiento se aborda desde un ámbito objetivo en tanto acontece en un horizonte gnoseológico de comprensión de lo real. Esta es la base del pensamiento suareciano desde donde se ilumina la tradición heredada. Hasta aquí el supuesto que ha colocado las premisas del presente trabajo.

Ahora bien, en concordancia con este supuesto, se ha buscado subrayar, a modo de introducción, los criterios fundamentales sobre los que el Dr. Eximio concibe su división de las ciencias especulativas de acuerdo con las $D M$ I. La proximidad y fidelidad del teólogo jesuita a Tomás de Aquino es ciertamente incorrecta, ya porque la clave de interpretación para el dominico está en el genus-subiectum de cada ciencia, ya porque las ciencias no dependen de la operación intelectual de abstracción, ya porque el subiectum es la manifestación de la primacía del actus essendi en clave científica. Sobre estas diferencias, las similitudes entre ambos órdenes de ciencias son externas.

De acuerdo con el pensamiento de Francisco Suárez, el ámbito especulativo señalaría la asunción intelectual de la naturaleza, de modo tal que la noción de filosofía expresaría la totalidad del saber, mientras que aquella de filosofía de la naturaleza se reduciría y equivaldría a la expresión ciencia especulativa, cuyo fin es la contemplación de la verdad. A su vez este fin se cumple en cada ciencia genérica y en cada ciencia específica que le queda sujeta. La relación de dependencia esencial y no secundum esse entre las ciencias genéricas del ámbito especulativo es como sigue: a. según el criterio quoad nos y de modo ascendente; b. según el criterio perse y de modo descendente. A su vez el primero se divide en: a.1. ciencia física; a.2. ciencia matemática; a.3. ciencia metafísica, filosofía primera o teología natural. Empero, si se atiende al segundo criterio, la división es la siguiente: b.1. ciencia metafísica, filosofía primera o teología natural; b.2. ciencia física; b.3. ciencia matemática. A su vez, la ciencia física se divide en: a. ciencia geológica; b. ciencia astronómica; c. ciencia biológica, cuya culminación es la ciencia de la antropología. Por su parte, la ciencia matemática presenta la siguiente división: a. aritmética; b. geometría; c. música (al depender de los principios de la aritmética); d. perspectiva (al depender de los principios de la geometría). 


\section{Bibliografía}

\section{Fuentes}

\section{Ediciones}

»Francisco Suárez, Disputationes Metaphysicae. Digitalisierungsprojekt - koordiniert von Prof. Salvador Castellote und Dr. Michael Renemann. Disponible en la web: http://homepage.ruhr-uni-bochum.de/Michael.Renemann/suarez/

\section{Traducciones}

" Francisco Suárez (1960). Disputaciones Metafísicas. Ed. y trad. Rábade Romeo, S., Caballero Sánchez, S. y Puigcerver Zanón, A. Madrid: Gredos. (Biblioteca Hispánica de Filosofía).

» Francisco Suárez (2011). Disputaciones metafísicas. Presentación de Rábade Romeo S.; Estudio preliminar de Florido, F. L. Madrid: Tecnos.

\section{Bibliografía complementaria}

" Alejo Montes, F. J. (1990). "La Universidad de Salamanca en el s. XVI: la reforma educativa de D. Juan de Zuñiga (1594)", Studia histórica: Historia Moderna 8, 151162.

» Bonino, S.-T. (1997). "Avant-propos. Le thomisme et son historie", Revue thomiste $97,5-8$.

" Bonino, S.-T. (2000). "L'école thomiste au XVe siècle", Rivista Teologica di Lugano V.2, 223-234.

"Bonino, S.-T. (2007). "Le thomisme parisien au XVe siècle", Revue thomiste 107,625-653.

» Courtine, J.-F. (2015). Suarez et le système de la métaphysique. París: Épiméthée.

"Cuccia, E. (2015). “El problema tomístico de la abstracción. Una reconsideración en torno a la interpretación tradicional y sus limitaciones", Cauriensia X, 245-263.

"Cuccia, E. (2017a). "El sentido de la abstracción en Tomás de Aquino. Una revisión a partir del uso textual del término", Scripta Mediaevalia 10.1, 83-112.

» Cuccia, E. (2017b). "Abstracción y matemática en el Comentario a la Física de Tomás de Aquino: más allá de las operaciones intelectuales", Eidos 27, 154-173.

» Cuccia, E. (2018). "La abstracción en Tomás de Aquino: una vía más allá de la epistemología tomista", Areté. Rev. de Filosofía 30.2, 245-263.

» Fabro, C. (1965). Historia de la Filosofía I. Madrid: Rialp.

»Florido, F. L. (2010). Las filosofías en la Edad Media. Crisis, controversias y condenas. Madrid: Biblioteca Nueva.

» Gilson, E. y Langan, Th. (1963). Filosofía Moderna. Barcelona: Emecé.

» Gómez López, C. (1996). “La Renovatio Urbis: poder, ciudad y universidad en el s. 
XVI", Espacio, Tiempo y Reforma. Serie VII. Historia del Arte 9, 53-76.

» Hale, J. R. (2016). La Europa del Renacimiento 1480-1520. Madrid: Siglo XXI.

» León Florido, F. L. (2000). "Una estructura filosófica en Historia de la Filosofía”, Anales del Seminario de Historia de la Filosofía 17, 195-216.

» León Florido, F. (2011). "Estudio Preliminar". En: Suárez, F. Disputaciones metafísicas. Madrid: Tecnos.

» Lohr, Ch. H. (1996). "Del aristotelismo medieval al aristotelismo renacentista”, Patristica et Mediaevalia 17, 3-15.

" Lorenz, D. (2003). "Sobre algunos disensos, progresos y crisis en la historia de la metafísica tomista", Revista Philosophica 26, 1-16.

» Mendoza, J. M. F. (2015). “Breve status quaestionis de la ciencia neotomista como introducción confusa al sentido de scientia tomística: algunas apreciaciones históricas en filósofos del siglo XX", Pensamiento 71.267, 617-630.

» Mendoza, J. M. F. (2017). La noción de scientia en Tomás de Aquino. Un estudio acerca de los sujetos de las ciencias, las distinciones de sus nombres, sus divisiones y ordenamiento. Buenos Aires: Prometeo.

» Muñoz, C. P. D. (2016). Objetividad y ciencia en Cayetano. Una prefiguración de la Modernidad. Santiago de Chile: CET.

» Parker, G. (2017). Europa en crisis 1598-1648. Madrid: Siglo XXI.

» Prieto López, L. (2013). Suárez y el destino de la metafísica, Madrid: BAC.

" Rodríguez-San Pedro de Bezares, L. E. (1986). La Universidad Salmantina del Barroco, período 1598-1625. T. II: régimen docente y atmósfera intelectual. España: Universidad de Salamanca.

» Verdú Berganza, I. (1993). "Aspectos generales del pensamiento en el siglo XIV", Anales del Seminario de Historia de la Filosofía 10, 195-208. 
